\title{
Leptospira interrogans en una población canina del Gran Buenos Aires: variables asociadas con la seropositividad ${ }^{1}$
}

\author{
Diana Rubel, ${ }^{2}$ Alfredo Seijo, ${ }^{3}$ Beatriz Cernigoi, ${ }^{3}$ Alberto Viale ${ }^{3}$ y \\ Cristina Wisnivesky-Colli ${ }^{2}$
}

RESUMEN Se determinó la seroprevalencia de leptospirosis en una población canina suburbana con el objeto de analizar la asociación entre distintas variables individuales y ambientales y la seropositividad a leptospirosis. El estudio, de diseño transversal, se llevó a cabo durante julio de 1992 en un barrio del Gran Buenos Aires en el que viven unos 9500 habitantes y una población canina de unos 2000 animales. Se estudió una muestra aleatoria de 223 perros, de cada uno de los cuales se obtuvo una muestra de sangre. La ficha epidemiológica del animal se obtuvo por encuesta al ama de casa. Las determinaciones serológicas se realizaron por microaglutinación frente a 10 serotipos de Leptospira interrogans. Se halló seropositividad en 57\% de los 223 perros examinados; $82 \%$ de los sueros positivos coaglutinaron con dos o más serotipos. Los serotipos detectados con mayor frecuencia fueron canicola y pyrogenes. La seroprevalencia en hembras fue menor que en machos $(\mathrm{P}<0,05)$ y entre los cachorros de menos de 1 año de edad, menor que en los animales de mayor edad ( $\mathrm{P}<0,01)$. El callejeo del perro y la presencia de agua estancada frente a la vivienda del propietario fueron los factores de riesgo más importantes entre los que se estudiaron. Las asociaciones de la seropositividad con el contacto con un basural, con el comportamiento de caza del perro y con la presencia de roedores en la vivienda no fueron estadísticamente significativas. Se discuten distintas medidas de control.

La leptospirosis es una zoonosis de amplia distribución con una variedad de reservorios, entre ellos muchas especies de mamíferos domésticos y silvestres. El conocimiento de las variables relacionadas con la adquisición de la infección en los animales tiene gran importancia para el diseño de políticas de control a nivel local. Los roedores se consideran el principal

1 Estudio financiado por la Secretaría de Ciencia y Técnica de la Universidad de Buenos Aires.

2 Universidad de Buenos Aires, Facultad de Ciencias Exactas y Naturales. Dirección postal: Ciudad Universitaria, Pab. 2, $4^{\circ}$ piso, CP 1428, Buenos Aires, Argentina. E-mail: paras@biolo.bg.fcen.uba.ar.

3 Hospital Francisco J. Muñiz, Servicio de Zoonosis, Buenos Aires, Argentina. reservorio, pero los perros podrían tener una importancia epidemiológica similar debido a su estrecha asociación con el hombre $(1,2)$. Los perros pueden transmitir la leptospirosis por contacto directo (al hombre $\mathrm{u}$ otros animales) o por contaminación del ambiente (agua o suelo) $(1,3)$.

En estudios serológicos realizados en distintos ambientes urbanos del continente americano, generalmente con perros vagabundos, se han hallado seroprevalencias de 18 a $63 \%$ (4-7). En Buenos Aires, estudios previos sobre leptospirosis canina han revelado prevalencias serológicas de 10 a $60 \%$ (8-11), siendo los serotipos canicola e icterohaemorrhagiae los detectados con mayor frecuencia.

Los objetivos del presente estudio fueron estimar la seroprevalencia de leptospirosis en una población canina suburbana, así como determinar la reactividad frente a los distintos serotipos y analizar los factores epidemiológicos asociados con la seropositividad.

\section{MATERIALES Y MÉTODOS}

\section{Área de estudio}

El estudio se llevó a cabo en San Nicolás, barrio ubicado en el munici- 
pio Florencio Varela del Gran Buenos Aires, donde las condiciones sociales y ambientales son características de gran parte de la zona. El barrio abarcaba en la época del estudio un área de aproximadamente 100 hectáreas y su población residente era de unas 9500 personas según el Censo Nacional de 1990. Las condiciones de saneamiento eran precarias: no existía servicio de agua potable, ni sistema cloacal. La mayor parte de las calles eran de tierra, muchas de ellas limitadas lateralmente por zanjas con aguas estancadas. Adyacente al barrio había un basural clandestino de 4 hectáreas de extensión, que recibía residuos de distinto origen (doméstico, veterinario, etc.).

La población canina domiciliada en el área era de 1940 animales según una evaluación demográfica preliminar (datos inéditos). Había al menos un perro en $72 \%$ de las casas. En cuanto al perfil sanitario de la población, $71 \%$ de los animales no habían recibido ningún control veterinario $y$ $29 \%$, solamente uno. No se había administrado ninguna vacuna a $45 \%$ de los perros y $42 \%$ solo habían recibido la antirrábica durante alguna de las campañas domiciliarias gratuitas realizadas por el municipio.

\section{Diseño del estudio}

El tamaño muestral se estimó sobre la base de los resultados de un muestreo preliminar. El estudio se llevó a cabo durante el mes de julio de 1992, previa difusión de sus objetivos en la comunidad. Se eligió el período de menor temperatura y precipitación del año, en el supuesto de que sería el de menor incidencia de leptospirosis. Para la selección al azar de los domicilios se numeraron las manzanas, cuadras y viviendas y posteriormente se aplicó una tabla de números aleatorios. Se incluyeron todos los perros residentes de 3 meses de edad o mayores, previo consentimiento de sus dueños. Ninguno de los perros había sido vacunado contra la leptospirosis.

La información epidemiológica que se analizó se obtuvo mediante una encuesta a las amas de casa. Las pre- guntas fueron abiertas y para la mayor parte de las variables había preguntas redundantes, a fin de aumentar el nivel de fiabilidad de las respuestas. Las variables se clasificaron posteriormente en categorías descriptivas de diferentes niveles de exposición. Se obtuvieron sueros de un total de 223 perros, con sus correspondientes cuestionarios epidemiológicos de 153 viviendas. La muestra incluyó $8,5 \%$ de las casas y $11,5 \%$ de los perros domiciliados en el área de estudio.

\section{Obtención y análisis de los sueros}

Las muestras de sangre se obtuvieron por venopuntura y el suero extraído se conservó a $-20{ }^{\circ} \mathrm{C}$ hasta su examen serológico. La presencia de anticuerpos se detectó por medio de microaglutinación $(11,12)$. Se usaron como antígenos los siguientes serotipos: icterohaemorrhagiae, canicola, pyrogenes, tarassovi, ballum, grippotyphosa, hebdomadis, hardjo, pomona y wolfii. Un suero se consideró seropositivo cuando el título (es decir, el inverso de la máxima dilución microaglutinante) fue de 100 o más para algún serotipo.

\section{Análisis estadístico}

Para determinar la asociación entre seropositividad y cada una de las variables analizadas se utilizó la prueba de $\chi^{2}$ (ji cuadrado) (13) y para analizar si existía una asociación tendencial entre la proporción de positivos y diferentes grados de exposición se utilizó la prueba $\chi^{2}$ de tendencia de Mantel-Haenszel (14).

\section{RESULTADOS}

El porcentaje de animales seropositivos fue $57 \% \pm 6(P<0,05)$. De los 127 sueros positivos, $104(82 \%)$ coaglutinaron con dos o más serotipos. El patrón de coaglutinación más frecuente fue canicola-pyrogenes, asociación que se halló en 88 (69\%) de los 127 sueros positivos. Los sueros que aglutinaron con cada serotipo fueron los siguien- tes: pyrogenes 114, canicola 109, icterohaemorrhagiae 41, ballum 16, hebdomadis 15, hardjo 14, tarassovi 12, grippotyphosa 11, pomona 3 , wolfii 0 . Los títulos máximos de microaglutinación para cada suero variaron entre 100 y 12800 , con una mediana de 200.

A partir del análisis de la encuesta se definieron las categorías de exposición y se calcularon las seroprevalencias correspondientes (cuadro 1).

La seroprevalencia global resultó significativamente mayor $(P<0,05)$ en machos $(63 \%)$ que en hembras $(45 \%)$, pero esta relación no se verificó en los animales menores de 1 año (cuadro 2). En este grupo de edad, la seroprevalencia fue significativamente menor $(12 / 36=33 \%)$ que en los perros de 1 año o más $(107 / 173=62 \%, P<0,01)$.

La seroprevalencia de los perros con vida callejera (una o más salidas diarias) fue significativamente mayor $(119 / 200=59 \%)$ que la de los perros confinados en la vivienda $(8 / 22=36 \%$, $P<0,05$, cuadro 1$)$. De estos últimos, $45 \%$ eran cachorros de menos de 1 año de edad. En el grupo de animales de menos de 3 años de edad la seropositividad se incrementó linealmente con el grado de contacto con la calle $(P<0,05$, niveles $0 ; 1-2 ; 3$ del cuadro 1 ). Además, en el grupo de perros más callejeros (más de una salida diaria a la calle), la seroprevalencia fue significativamente mayor entre los animales de viviendas con zanja en la misma cuadra de la vivienda $(79 / 123=64 \%)$ que en los de viviendas sin zanjas en sus cercanías $(8 / 22=36 \%, P<0,05)$. La seropositividad se incrementó linealmente con la presencia transitoria y permanente de zanja frente a la casa $(P<0,05)$.

La asociación de la seropositividad con el contacto del perro con el basural no fue estadísticamente significativa $(P=0,06)$, aunque la proporción de seropositivos fue menor entre los perros sin contacto con él (52\%) que entre los que lo visitaban $(66 \%$, cuadro 1). Entre los perros "cazadores", la seroprevalencia fue mayor $(67 \%)$ que entre los "no cazadores" (54\%), pero la diferencia tampoco fue estadísticamente significativa $(P=0,1)$. Un tercio de los perros "cazadores" habían sido observados cazando roedores. 
CUADRO 1. Niveles de exposición y porcentaje incluido en cada nivel, del total de 223 perros de una muestra de la población canina del barrio de San Nicolás, municipio Florencio Varela, Gran Buenos Aires, 1992

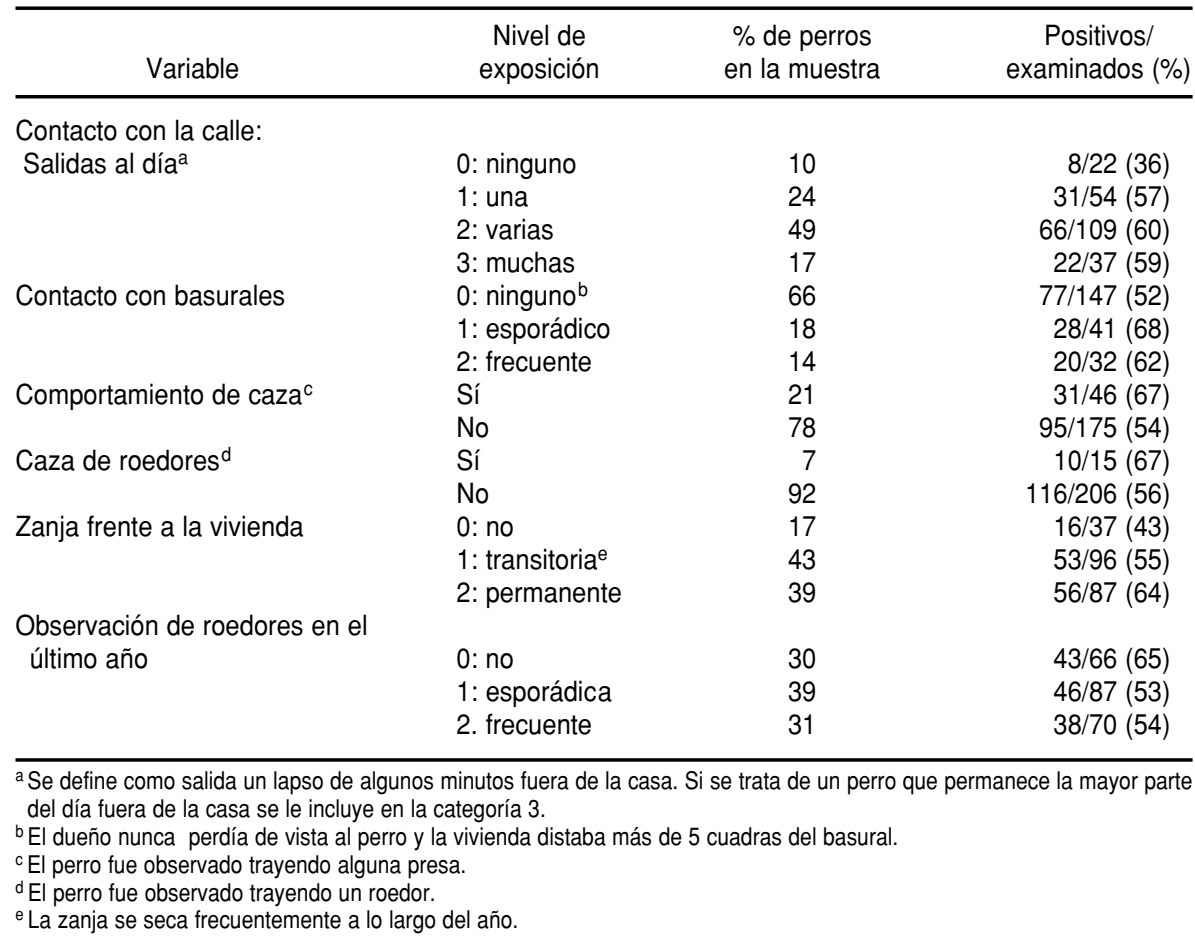

El nivel de roedores en la casa tampoco se asoció con seropositividad $(P=0,27)$, al igual que la presencia de otros animales domésticos $(P=0,26)$. El análisis de asociación tampoco reveló diferencias estadísticamente significativas cuando se realizó para cada especie de animal doméstico presente. En el área se criaban gatos $(80 \%$ de las casas), gallinas (37\%), conejos $(9 \%)$, cerdos $(8 \%)$, loros $(7 \%)$, pájaros (6\%) y caballos (5\%).

Al relacionar los datos de la encuesta con los resultados serológicos se observó que 23 de los 127 perros seropositivos y 17 de los 96 seronegativos presentaron algún síntoma durante el último año $(P=0,9)$. Algunos de los síntomas solo fueron mencionados por los dueños para perros seropositivos (10 animales en total): decaimiento con pérdida de peso, decaimiento con vómitos o falta de apetito, vómitos con sangre, diarrea con vómitos.

CUADRO 2. Seropositividad a leptospirosis según sexo y edad en una muestra de perros del barrio de San Nicolás, municipio Florencio Varela, Gran Buenos Aires, 1992

\begin{tabular}{lrrr}
\hline \multirow{2}{*}{$\begin{array}{c}\text { Edad } \\
\text { (años) }\end{array}$} & \multicolumn{3}{c}{ Positivos/examinados (\%) } \\
\cline { 2 - 4 } & \multicolumn{1}{c}{ Machos } & Hembras & \multicolumn{1}{c}{ Total } \\
\hline$<1$ & $5 / 18(28)$ & $7 / 18(39)$ & $12 / 36(33)$ \\
1 & $13 / 18(72)$ & $7 / 13(54)$ & $20 / 31(64)$ \\
2 a 3 & $28 / 35(80)$ & $9 / 19(47)$ & $37 / 54(68)$ \\
4 a 5 & $12 / 21(57)$ & $7 / 17(41)$ & $19 / 38(50)$ \\
6 a 7 & $11 / 16(69)$ & $2 / 5(40)$ & $13 / 21(62)$ \\
8 a 12 & $16 / 26(61)$ & $2 / 3(67)$ & $18 / 29(62)$ \\
Total & $85 / 134(63)$ & $34 / 75(45)$ & $119 / 209^{a}(57)$ \\
\hline
\end{tabular}

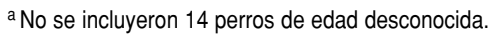

\section{DISCUSIÓN}

La prevalencia de animales seropositivos indica un alto nivel de circulación de Leptospira interrogans en la población canina del área. Los patrones de positividad obtenidos y la escasa observación de síntomas podrían reflejar, como sugiere un estudio similar, ${ }^{4}$ una situación de estimulación antigénica persistente en un área de alta endemicidad donde las infecciones asintomáticas son frecuentes. Apoya esta hipótesis la ausencia de síntomas en el único animal cuyo suero presentó un título de 12800.

La endemicidad del área se infiere tanto del máximo que alcanza la seroprevalencia al año de edad como de la alta frecuencia de exposición de los animales a los factores de riesgo estudiados.

Nuestro estudio aporta pruebas de que el contacto con la calle es el factor de riesgo más importante para la población canina. Esto podría deberse a que en las calles del área estudiada se hallan zanjas y otros microambientes favorables para la supervivencia de la leptospira. Fue común observar que los perros bebían, orinaban y caminaban dentro de las zanjas, y dado que esas zanjas también suelen ser frecuentadas por roedores, podrían ser un elemento importante en la transmisión por contaminación ambiental. Sin embargo, no puede descartarse la existencia de transmisión directa entre perros, o entre perros y roedores. Es probable que las calles sean los sitios donde se producen con mayor frecuencia los contactos entre estos animales.

Se ha sugerido que el serotipo canicola es particularmente frecuente en las áreas urbanas en que se producen contactos habituales entre perros vagabundos (2). Dadas las características ambientales y los hábitos de manejo de la población canina en este tipo de área, la categoría "perro vagabundo" sería epidemiológicamente equiva-

4 Pereira MM. Leptospirose em área urbana do Municipio do Rio de Janeiro [tesis de maestría]. Rio de Janeiro: Instituto Oswaldo Cruz-Fiocruz; 1985. 
lente a la de "perro con dueño", ya que la mayoría de los perros tienen acceso a las calles, no usan ninguna identificación y carecen de atención veterinaria.

El confinamiento de los animales en las viviendas o la vacunación masiva serían medidas de control posibles, pero ambas resultarían de difícil implementación y seguimiento. Medidas de control ambiental, principalmente el asfaltado de las calles y la instalación de alcantarillado, podrían disminuir la prevalencia en la poblaSulzer CR. Leptospirosis in dogs and cats on the island of Trinidad, West Indies. Int $J$ Zoonoses 1979;6:33-40.

2. Everard COR, Jones CJ, Inniss VA, Carrington DG, Vaughan AW. Leptospirosis in dogs in Barbados. Isr J Vet Med 1987;43(4):289-295.

3. Faine S. Guidelines for control of leptospirosis. Geneva: World Health Organization; 1982. (WHO Offset Publication 67).

4. Thiermann AB. Canine leptospirosis in Detroit. Am J Vet Res 1980;41(10):1659-1661.

5. Farrington NP, Sulzer KR. Canine leptospirosis in Puerto Rico. Int I Zoonoses 1981:9:45-50.

6. Tsuneshigue Fukuda CT, Heysen MA, Liceras de Hidalgo J. Epidemiología de la leptospirosis canina en la provincia del Callao-1971. Rev Inst Zoonosis Invest Pecuar (Lima) 1973;II $(1,2): 6-28$.

7. Pereira MM, Andrade J. Human leptospirosis in a slum area in the city of Rio de Janeiro, ción canina y tener mayor efecto desde el punto de vista de la salud pública.

El Gran Buenos Aires es la zona de la Argentina con la mayor notificación de casos de leptospirosis humana y $41 \%$ de los pacientes atendidos de 1984 a 1994 presentaron serorreactividad al serotipo canicola (15). Dado que la zona del estudio presenta condiciones ambientales y socioeconómicas típicas para toda la zona, los resultados del estudio sugieren que el

\section{REFERENCIAS}

Brazil: a serological and epidemiological study. Mem Inst Oswaldo Cruz 1990;85(1):47-52.

8. Savino E, Rennella E. Estudios sobre leptospirosis: III. Presencia de leptospiras en los perros de la ciudad de Buenos Aires. Rev Inst Bacteriol Malbrán (Buenos Aires) 1944;12: 215-226.

9. Cacchione RA, Cedro VC, Bulgini MJ, Cascelli ES, Martínez ES. Leptospirosis canina en la República Argentina: difusión, morbilidad. Rev Invest Ganad (Buenos Aires) 1962;14: 125-132.

10. Myers DM. Leptospiral antibodies in stray dogs of Moreno, Province of Buenos Aires. Rev Argent Microbiol 1980;12(1):18-22.

11. Asociación Argentina de Veterinarios de Laboratorios de Diagnóstico, Comisión Científica Permanente sobre Leptospirosis. Manual de leptospirosis. AAVLD; Buenos Aires: 1994.

12. Myers D. Leptospirosis: manual de métodos para el diagnóstico de laboratorio. Buenos perro podría ser una fuente de infección importante para la leptospirosis humana.

Agradecimiento. La investigación fue posible gracias a la participación y apoyo de numerosos vecinos de la zona de estudio, especialmente Susana Viroche y su familia. Los autores agradecen también los comentarios de Silvia Pietrokovsky a la versión inicial del texto.
Aires: Centro Panamericano de Zoonosis, OPS/OMS; 1985. (Nota técnica 30).

13. Fleiss JL. Statistical methods for rates and proportions, 2a ed. New York: John Wiley; 1981.

14. Schlesselman JJ. Case-control studies. New York: Oxford University Press; 1987.

15. Asociación Argentina de Zoonosis. Primer Congreso Argentino y Primer Congreso Latinoamericano de Zoonosis: resumen G2. Buenos Aires: AAZ; 1995.

Manuscrito recibido el 26 de enero de 1996 y aceptado para publicación en versión revisada el 11 de septiembre de 1996

ABSTRACT We determined the seroprevalence of leptospirosis in a suburban canine population for the purpose of analyzing the association between different individual and environmental variables and seropositivity for leptospirosis. The study, which was crosssectional, was performed in July 1992 in a neighborhood of Greater Buenos Aires with approximately 9500 inhabitants and a canine population of around 2000 animals. We studied a random sample of 223 dogs and obtained a blood sample from each. Each animal's epidemiologic history was obtained by interviewing the housewife. Serologic measurements were performed by the microagglutination technique with the use of 10 different serotypes of Leptospira interrogans. Of the 223 dogs that were tested, $57 \%$ showed seropositivity; $82 \%$ of the positive sera coagglutinated with two or more serotypes. The most frequently detected serotypes were canicola and pyrogenes. Seroprevalence in females was less common than in males $(P<0,05)$ and in puppies less than 1 year old it was less common than in older animals $(P<0,01)$. Street behavior in the dog and the presence of stagnant water in front of the owner's dwelling were the most important of the risk factors examined. The associations between seropositivity on the one hand and contact with trash deposits, hunting behavior and the presence of rodents inside the dwelling on the other were not statistically significant. Different control measures are discussed. 


\section{ERRATA}

Nascimento, et al., "Cobertura do teste de Papanicolaou

no município de São Paulo e características

das mulheres que realizaram o teste"

(Boletín de la Oficina Sanitaria Panamericana 1996;121(6):491-501)

Chamamos a atenção dos leitores para os seguintes erros, que são de total responsabilidade de nossa redação:

- P. 495: A terceira e quarta frases do primeiro parágrafo do texto devem ser substituídas por "Cabe notar, contudo, que após ajuste, os ORs das faixas etárias de 25 a 34 e 35 a 44 anos, de história de uso de contraceptivo oral e realização de auto-exame de mama foram menores do que na análise univariada".

- P. 496: A segunda frase do parágrafo anterior à "Discussão" deve ser substituída por "A comparação desses resultados com aqueles observados quando a realização do Papanicolaou alguma vez na vida é a variável dependente mostra aumento dos ORs para todas faixas etárias (45-59 anos como referência) e redução dos 0Rs nas mulheres que já usaram contraceptivo oral e nas que relataram auto-exame de mama".

- P. 499: A segunda e terceira frases devem ser substituídas por "Contudo, foi observado um aumento nos ORs em todas as faixas etárias e redução dos ORs nas mulheres que já haviam usado contraceptivo oral e nas que relataram auto-exame de mama. No tocante à faixa etária (referência 45-59 anos), essas diferenças devem refletir, ao menos em parte, a maior probabilidade de que as mulheres jovens, quando colhem a citologia, fazem 0 exame em época recente (nos últimos 3 anos). Ou, de modo análogo, as mulheres de 45-59 anos teriam colhido Papanicoulaou há mais de 3 anos".

\section{$\bullet \bullet \bullet$ \\ Nascimento, et al., "Pap Test Coverage in São Paulo Municipality and Characteristics of the Women Tested" (Bulletin of the Pan American Health Organization 1996;30(4):302-312)}

We beg our readers to note the following errors, which are entirely the responsibility of our editorial staff:

- Pp. 306-307: The last sentence on p. 306 should read "It should be noted, however, that following adjustment the ORs for the 25-34 and 35-44 age groups corresponding to use at some time of oral contraceptives and having performed breast self-examination, were smaller (relative to the $45-59$ group) than in the univariate analysis."

- P. 308: The second sentence should read "Comparison of these results with those when the Pap test was taken sometime in the past showed an increase in the ORs for all age groups (relative to the 45-59 group) and a decline in the ORs among women who had used oral contraceptives and women who reported performing breast self-examination."

- Pp. 310-311: The last sentence on p. 310 should read "However, an increase in the ORs was observed among all age groups and a reduction was noted in the ORs for women reporting use of oral contraceptives and performance of breast self-examinations. With regard to age group (using as reference women 45-59), these differences should reflect, at least partially, the greater likelihood that young women who have undergone cytology testing have done so recently (in the last three years). Similarly, women aged 45-59 would be more likely to have been tested more than three years before." 